\title{
Are prolific authors too much of a good thing?
}

The last sentence in the first paragraph of this Editorial contains an error (BMJ 2015;351:h2782, doi:10.1136/bmj.h2782). This sentence should have read: "In a similar study of prolific authors, it was found that of the 40 most productive authors in four medical specialties, 24 were named on at least one publication per 10 working days each year, showing that the issue is not restricted to diabetes research."

Cite this as: BMJ 2015;351:h4667

๑ BMJ Publishing Group Ltd 2015 